\title{
Successful early diagnosis and treatment of non-convulsive status epilepticus-induced Takotsubo syndrome
}

\author{
Yusuke Morinaga*, Kouhei Nii, Kimiya Sakamoto, Ritsurou Inoue, Takafumi Mitsutake, \\ Hayatsura Hanada
}

Department of Neurosurgery, Fukuoka University Chikushi Hospital, Chikushino, Fukuoka, Japan.

SUMMARY Takotsubo syndrome (TTS) is often preceded by emotional or physical stress. Epileptic seizures have been described in more than 100 TTS cases. Due to the lack of typical symptoms, seizureinduced TTS can be overlooked. Here, we describe a rare case where TTS induced by nonconvulsive status epilepticus (NCSE) was diagnosed early and successfully treated. An 82-yearold man presented to our hospital with confusion, anorexia, aphagia, and abnormal behavior beginning a few days earlier. Head computed tomography and magnetic resonance imaging did not show any structural abnormalities. Upon hospitalization, blood sampling revealed elevated levels of myocardial escape enzymes; however, cardiac ultrasonography showed apical asystole, and emergency coronary angiography did not show any significant stenosis or occlusion. The patient's symptoms improved after the administration of antiepileptic drugs consisting of diazepam, fosphenytoin, and levetiracetam. On day 2 of hospitalization, an electroencephalogram showed high amplitude slow waves in the left cerebral hemisphere and NCSE-induced TTS was diagnosed. The patient was discharged after 2 weeks with a modified Rankin Scale score of 0 and continuing oral administration of levetiracetam. Delay in the diagnosis of NCSE-induced TTS can lead to a poor prognosis. Early diagnosis and treatment for NCSE and NCSE-induced TTS may result in favorable outcomes for the patient.

Keywords non-convulsive status epilepticus, Takotsubo syndrome, trazodone

\section{Introduction}

Takotsubo syndrome (TTS) is often preceded by emotional or physical stress. Epileptic seizures have been described in more than 100 TTS cases.. Due to the lack of typical symptoms, seizure-induced TTS can be overlooked (1). Specifically, there are few reports of non-convulsive status epilepticus (NCSE)-induced TTS (2). NCSE (3) is common in the elderly. It most often involves prolonged focal seizures with impaired contact, known as complex partial status epilepticus. A form of de novo absence status epilepticus can also occur, which is much rarer. The identified risk factors for the onset of NCSE are precession by a generalized tonic-clonic seizure, a known history of epilepsy, female gender, and known brain injury (especially a stroke sequela). The presence of one of these risk factors combined with a confusional clinical symptom of unknown origin should lead us to consider a diagnosis of NCSE. As the clinical presentation is often quite general, for example involving stupor, confusion, and even coma, the diagnosis is based on electroencephalography (EEG) with validated criteria known as the Salzburg EEG criteria. The treatment first involves injection of benzodiazepines and then, intravenous, oral, or gastric tube administration of antiepileptic drugs. Intubation/ventilation are not generally recommended, except when absolutely necessary for example under respiratory distress and multi-organ failure (1). The prognosis is generally poor with approximately $30 \%$ mortality $(1)$.

Here, we describe a rare case where TTS induced by non-convulsive status epilepticus (NCSE) was diagnosed early and successfully treated.

\section{Case Report}

An 82-year-old man presented to our hospital with confusion, anorexia, aphagia, vomiting, and abnormal behavior beginning a few days earlier. He had history of hypertension but did not use any antihypertensive medication. Neurological assessments revealed a Japan Coma Scale grade 3, aphagia, and no limb paralysis. 
Upon hospitalization, his heart rate was $100 \mathrm{bpm}$ and his blood pressure was $169 / 102 \mathrm{mmHg}$. Blood sampling revealed elevated levels of myocardial escape enzymes (troponin T $1.34 \mathrm{ng} / \mathrm{mL}$, creatine kinase $\mathrm{MB}$ $59.9 \mathrm{U} / \mathrm{L}$ ), but no other abnormalities were noted. An electrocardiogram showed an ST-T wave rise at V2, 3. However, a cardiac ultrasound displayed apical asystole and an emergency coronary angiography did not show any significant stenosis or occlusion (Figure 1A). Plain head computed tomography (Figure 1B) and magnetic resonance imaging (Figure 1C) showed no abnormalities. The patient's symptoms improved after the administration of the antiepileptic drugs diazepam, fosphenytoin, and levetiracetam.

On day 2 of hospitalization, EEG revealed high amplitude slow waves in the left cerebral hemisphere (Figure 2). The patient was diagnosed with NCSEinduced TTS and was discharged 2 weeks later with a modified Rankin Scale score of 0; he was advised to continue the oral administration of levetiracetam 1,000 $\mathrm{mg} /$ day.

Written informed consent was obtained from the patient for publication of this case report and the accompanying images, and the study design was approved by the appropriate ethics review board.

\section{Discussion}

To the best of our knowledge, reports of successful early diagnosis and treatment for NCSE-induced TTS, such
(A)

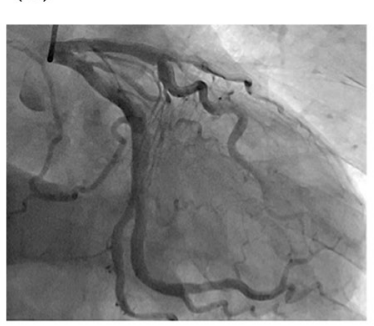

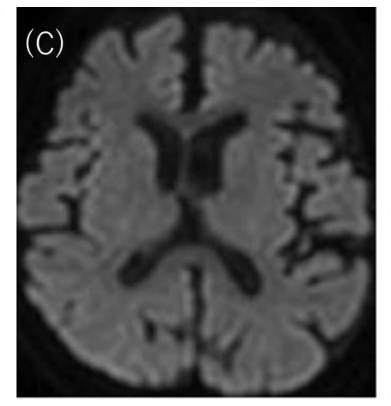

(D)

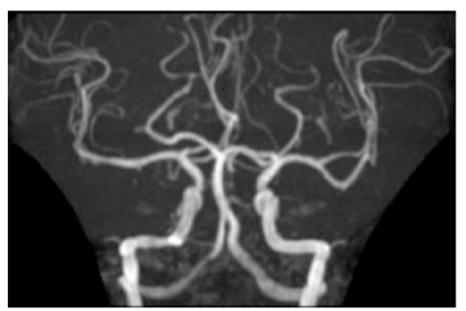

Figure 1. Clinical imaging at hospitalization. (A) Coronary angiography images acquired on day 1 of hospitalization do not show any significant coronary artery stenosis or occlusion. (B) Head plane computed tomography reveals no abnormalities. (C) Diffusion-weighted imaging shows no abnormalities. (D) Magnetic resonance angiography reveals no aneurysms or blood vessel malformations.

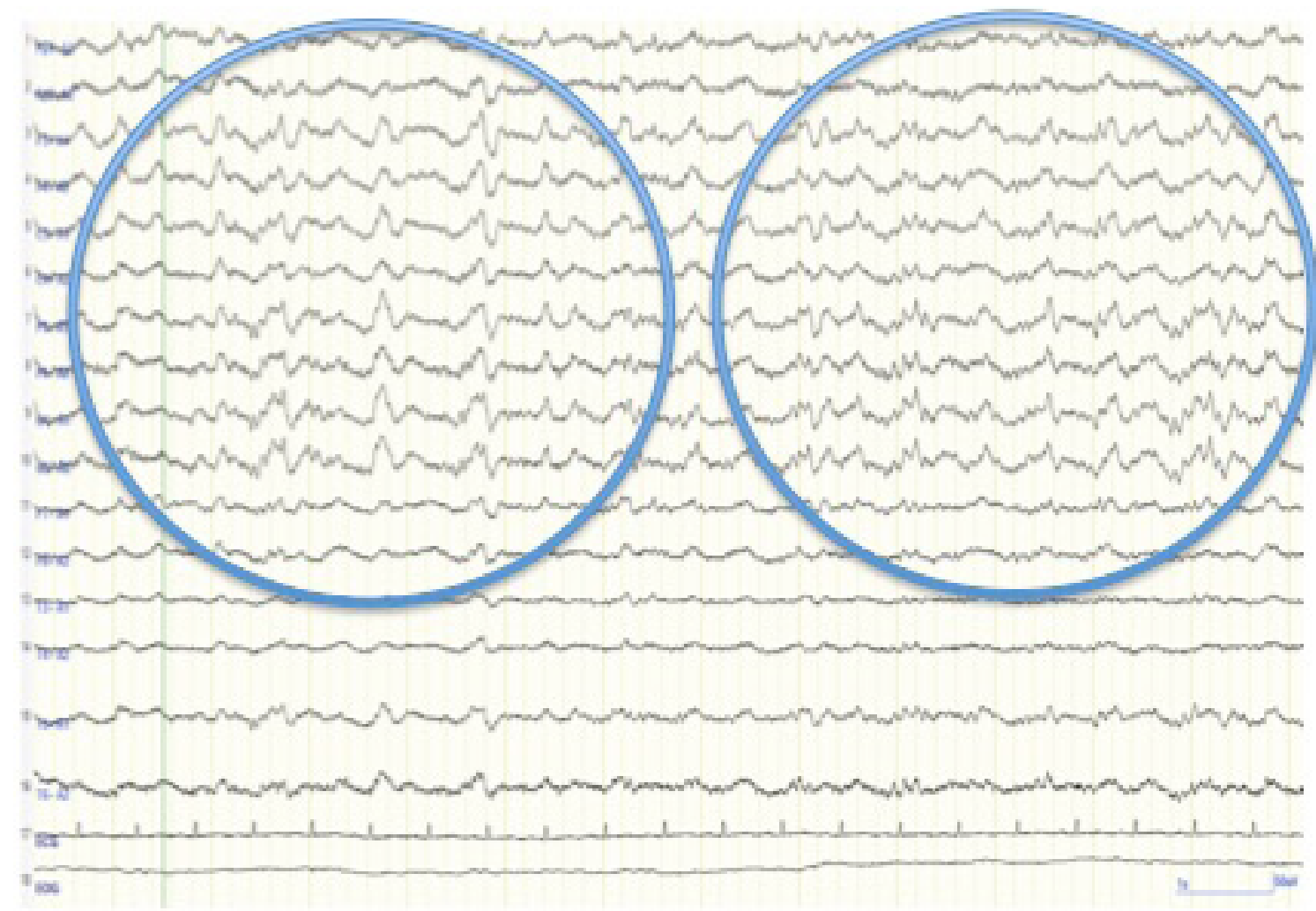

Figure 2. Examinations performed on day 2 of hospitalization. lectroencephalogram showing high amplitude slow waves in the left cerebral hemisphere. 
as the present one, are rare. Enhanced catecholamine production may cause TTS $(2,4)$. Catecholamines in the blood are elevated in diseases with high severity such as those involving convulsions and are implicated in the onset (2). Our case suggests that partial seizures may also be complicated. Uemura et al. (2) showed increased blood flow in the left temporal lobe and basal ganglia at the time of the seizures by single-photon emission computed tomography, and a high signal area in the left thalamus by head magnetic resonance diffusion weighted imaging. Based on these findings, they speculated that hyperactivation of neurocircuits may be associated with the onset of TTS (2).

Acute complications of takotsubo cardiomyopathy include pump ataxia, left ventricular apical thrombus formation, left ventricular outflow tract obstruction, and arrhythmia (torsade de pointes), each of which should be managed. Sudden death has been reported in cardiomyopathy cases (5), and takotsubo cardiomyopathy has been indicated as a cause of sudden death associated with epileptic seizures ( 6 ) and it has been reported that NCSE-induced takotsubo cardiomyopathy can result in sudden death (6). In takotsubo cardiomyopathy, chest pain is usually the main complaint, but in our case, there was no symptom of chest pain during the course. There is a possibility that consciousness disorder may influence the symptoms of NCSE-induced TTS. In our case, early diagnosis and subsequent treatment of NCSE-induced TTS enabled successful discharge of the patient within 14 days of admission.

In conclusion, delay in the diagnosis of NCSEinduced TTS can lead to a poor prognosis. Early diagnosis of NCSE and NCSE-induced TTS by clinical symptoms, elevated levels of myocardial escape enzymes (troponin $\mathrm{T}$ and creatine kinase $\mathrm{MB}$ ), electrocardiography, and EEG followed by a prompt and appropriate treatment may contribute to favorable clinical outcomes for the patient.

\section{Acknowledgements}

We would like to thank Editage (www.editage.com) for English language editing. This research did not receive any specific grants from funding agencies in the public, commercial, or not-for-profit sectors.

\section{References}

1. Stöllberger C, Sauerberg M, Finsterer J. Immediate versus delayed detection of Takotsubo syndrome after epileptic seizures. J Neurol Sci. 2019; 397:42-47.

2. Uemura J, Wada Y, Yagita Y. Non-convulsive status epilepticus with Takotsubo cardiomyopathy: a case report. Rinsho shinkeigaku. 2016; 56:852-856. (in Japanese).

3. Dupont S. Non convulsive status epilepticus in the elderly. Geriatr Psychol Neuropsychiatr Vieil. 2019; 17:25-30.

4. Nandal S, Castles A, Asrar U1 Haq MA, van Gaal W. Takotsubo cardiomyopathy triggered by status epilepticus: case report and literature review. BMJ Case Rep. 2019; 12:e225924.

5. Finsterer J, Bersano A. Seizure-triggered Takotsubo syndrome rarely causes SUDEP. Seizure. 2015; 31:84-87.

6. Stollberger C, Wegner C, Finsterer J. Seizure associated Takotsubo cardiomyopathy. Epilepsia. 2011; 52:e160-e167.

Received March 2, 2020; Revised May 9, 2020; Accepted May 13, 2020.

*Address correspondence to:

Yusuke Morinaga, Department of Neurosurgery and Neuroscience, Fukuoka University Chikushi Hospital, 1-1-1 Zokumyoin, Chikushino-shi, Fukuoka Prefecture 818-8502, Japan.

E-mail: yu_the_morio@yahoo.co.jp

Released online in J-STAGE as advance publication May 15, 2020. 